\title{
Voir et dire dans le Manuscrit trouvé à Saragosse
}

Jan Blanc

\section{CpenEdition}

Journals

Édition électronique

URL : http://journals.openedition.org/edl/458

DOI : $10.4000 /$ edl. 458

ISSN : 2296-5084

\section{Éditeur}

Université de Lausanne

\section{Édition imprimée}

Date de publication : 15 décembre 2012

Pagination : 97-114

ISBN : 978-2-940331-29-1

ISSN : 0014-2026

Référence électronique

Jan Blanc, « Voir et dire dans le Manuscrit trouvé à Saragosse », Études de lettres [En ligne], 4 | 2012, mis en ligne le 15 décembre 2015, consulté le 19 décembre 2020. URL : http://journals.openedition.org/ edl/458 ; DOI : https://doi.org/10.4000/edl.458 


\section{VOIR ET DIRE DANS LE MANUSCRIT TROUVÉ À SARAGOSSE}

Le Manuscrit trouvé à Saragosse $(1804,1810)$ de Jean Potocki regorge de références à l'histoire de l'art. Ces allusions offrent évidemment l'occasion à l'auteur polonais de faire valoir l'étendue d'une culture visuelle considérable. Mais elles lui permettent surtout, en construisant un univers visuel et onirique inédit, de servir les ambitions d'un projet poétique. En citant les œuvres de Raphaël et de Michel-Ange, les noms de Pacheco et de Velázquez, Potocki ne fait pas œuvre d'historien mais de mythographe qui, revisitant le monde de l'art ancien en fonction de situations et de personnages qu'il a lui-même imaginés, offre moins une fête pour l'œil qu'une célébration de la langue elle-même.

Pour un historien de l'art, dont les activités de lecture professionnelles sont plutôt liées au monde de l'image qu'à celui de l'écrit, lire le Manuscrit trouvé à Saragosse $(1804,1810)$ est, pour paraphraser Eugène Delacroix, une véritable fête pour l'œil ${ }^{1}$. Le travail de la langue qu'y déploie Jean Potocki (1761-1815) laisse en effet une large place aux métaphores et aux images, souvent frappantes, parfois inoubliables. Des images qui empruntent au registre littéraire et poétique comme aussi à celui de la peinture, de la gravure ou du théâtre, dont Potocki était visiblement un fin connaisseur.

Ce bref article propose d'esquisser une exploration de ces relations du texte et de l'image dans le Manuscrit. Les enjeux de l'intertextualité, si

I. E. Delacroix, Journal, 1822-1863, 22 juin 1863: «Le premier mérite d'un tableau est d'être une fête pour l'œil. Ce n'est pas à dire qu'il n'y faut pas de la raison: c'est comme les beaux vers [...]; toute la raison du monde ne les empêche pas d'être mauvais, s'ils choquent l'oreille. On dit: "avoir de l'oreille”; tous les yeux ne sont pas propres à goûter les délicatesses de la peinture. Beaucoup ont l'œil faux ou inerte; ils voient littéralement les objets, mais l'exquis, non.» 
essentiels à l'œuvre de Jean Potocki, ont fait l'objet, on le sait, de très nombreuses études, que cet article ne prétend pas remettre en cause ${ }^{2}$. Il est frappant de constater, toutefois, que les questions liées à la place et au rôle des artistes et des œuvres d'art cités dans le Manuscrit trouvé à Saragosse ont été assez largement ignorées, à l'exception des récits de voyage de Potocki, sur lesquels je reviendrai à plusieurs reprises ${ }^{3}$. Bien évidemment, il ne s'agit pas pour moi de renouveler une bibliographie que je suis loin de maîtriser, ni même de connaître de façon suffisamment satisfaisante. L'enjeu sera plutôt d'éclairer sous un jour particulier, de proposer une lecture personnelle, propre à ce qu'en peut livrer un historien de l'art, d'un texte fondamental de l'histoire littéraire du tout début du $\mathrm{XIX}^{\mathrm{e}}$ siècle, mais aussi d'un témoignage capital de ce que pouvait être la culture artistique d'un écrivain de la fin des Lumières. Ces quelques réflexions me permettront de faire état de quelques impressions personnelles sur le texte de Potocki, en parlant en historien de l'art, attaché, par l'habitude mais aussi par le goût personnel, aux œuvres, mais aussi à la vision, au visible, au visuel - autant de dimensions de l'expérience et des sens qui, me semble-t-il, constituent des thèmes centraux dans l'ouvrage de Potocki, qu'il serait utile d'éclairer davantage.

\section{L'oil et la langue}

A la première lecture du Manuscrit trouvé à Saragosse, il n'est guère difficile d'être frappé par les innombrables références, directes ou indirectes, que Jean Potocki y instille à l'histoire des arts européens. Ces références mériteraient probablement de faire l'objet d'un inventaire exhaustif, en se fondant sur une analyse minutieuse du texte et de ses sources, et en croisant cette analyse avec ce que l'on sait de la bibliothèque, des lectures et de la culture visuelle de Potocki. Je me contenterai ici d'en fournir quelques exemples, en commençant par les noms propres que l'auteur du Manuscrit convoque pour appeler les personnages de son roman.

2. Voir surtout J. Herman, P. Pelckmans, F. Rosset (éds), Le Manuscrit trouvé à Saragosse et ses intertextes, un recueil d'articles sur lequel je reviendrai à de multiples reprises.

3. F. Rosset, D. Triaire, De Varsovie à Saragosse, p. 111-124; S. Moussa, "Le nomadisme chez Potocki». 
Parmi ces patronymes, nombreux sont ceux qui sont familiers aux connaisseurs de la peinture occidentale, et notamment de la peinture espagnole, ce qui n'est guère étonnant dans le cadre d'un roman dont l'action se situe en grande partie dans la péninsule ibérique et qui ne cache pas ses hommages au Don Quichotte de Cervantès ${ }^{4}$. Lors de la dix-neuvième journée, dans la version de 1804, un long passage est ainsi consacré à l'histoire de don Pedre Velasquez, un géomètre dont le nom rappelle évidemment celui de Diego Velázquez, le grand peintre espagnol du Siglo de Oro (1599-1660) ${ }^{5}$. On nous explique d'ailleurs que le père de ce Velasquez, un certain don Enrique, avait été ingénieur pour le roi, ce qui a été également le cas du peintre espagnol, qui a occupé des fonctions similaires auprès du roi Philippe IV d'Espagne (1605-1665).

Un autre personnage, sans doute plus important dans l'économie narrative et symbolique du Manuscrit, fait lui aussi référence à la peinture du Siècle d'or espagnol. Ce personnage, à l'apparence difforme, apparait à Alphonse van Worden lors de la seconde journée, au matin de la nuit mystérieuse passée dans l'auberge de Venta Quemada, alors que le narrateur vient de faire la rencontre de l'ermite et d'entrer dans son logis:

Je vis entrer dans la cabane une figure plus effrayante que tout ce que j'avais vu jusqu'alors. C'était un homme qui paraissait jeune, mais d'une maigreur hideuse. Ses cheveux étaient hérissés; un de ses yeux était crevé et il en sortait du sang; sa langue pendait hors de sa bouche et laissait couler une écume baveuse. Il avait sur le corps un assez bon habit noir, mais c'était son seul vêtement; il n'avait même ni bas, ni chemise $^{6}$.

Le nom de cet être monstrueux, recueilli par l'ermite qui en fait son domestique, et soigné par le cabaliste don Pedre de Uzeda, est Pascheco, dans la version de 1804, ou Pacheco, dans celle de $1810^{7}$. Dans un cas comme dans l'autre, l'allusion est directe au peintre espagnol Francisco Pacheco (1564-1644), connu pour avoir écrit un célèbre traité de peinture (Arte de la pintura, su antigüedad y su grandeza, 1649), mais aussi, surtout, pour avoir été le maître de Diego Vélazquez.

4. L. Fraisse, Potocki et l'imaginaire de la création, p. 92.

5. MTS-1804, p. 337-358.

6. MTS-1804, p. 89.

7. MTS-1804, p. $90 ;$ MTS-1810, p. 89. 
Toutefois, le personnage de Pascheco n'offre pas seulement à Potocki l'occasion de faire l'étalage de sa culture picturale ou de jouer habilement avec les références et les citations. Cette créature occupe un rôle déterminant dans le Manuscrit trouvé à Saragosse, en illustrant métaphoriquement les rapports que Potocki entretient, en tant qu'écrivain, avec les images et les mots. Le personnage monstrueux de Pascheco se définit en effet par deux caractéristiques physiques: il est borgne («un de ses yeux était crevé et il en sortait du sang») et doté d'une langue démesurément longue ("sa langue pendait hors de sa bouche et laissait couler une écume baveuse»). Par un étrange effet de compensation, on a l'impression que la perte d'un de ses deux organes visuels a, chez Pascheco, favorisé le développement extravagant d'un des organes de son expression orale, dont le démoniaque fait d'ailleurs un excellent usage en ayant la «langue bien pendue» quand son maître lui ordonne, à deux reprises, de raconter sa propre histoire au narrateur ${ }^{8}$. Si la laideur de ce personnage est probablement liée à sa nature particulièrement diabolique ${ }^{9}$, elle me semble également relever, dans ses spécificités narratives, d'une réflexion sur l'imitation et les ressources respectives du langage visuel et oral.

Jean Potocki n'a jamais cessé de souligner, en effet, sa croyance dans les puissances créatrices du langage littéraire. Pour lui, il est clair qu'«il faut dire pour jouir " ${ }^{10}$, et que si les relations entre le regard et le discours sont réelles, ne serait-ce que parce que les fruits du premier peuvent inspirer ceux du second, le regard ne suffit jamais véritablement pour Potocki, qui ne cache pas cultiver un rapport logocentrique au monde et à la nature:

Je me souviens que, dans l'âge où l'esprit voudrait embrasser à la fois toutes les connaissances [...] j’aurais désiré lire, dans un été, tous les livres qui existaient alors sur l'histoire naturelle. [...] L'histoire des peuples de la haute Asie m’a beaucoup occupé, mais seulement dans les livres. Maintenant je les vois devant moi ces peuples, avec leurs traits caractéristiques, leurs ressemblances, leurs différences, leurs idiomes et leurs traditions ${ }^{11}$.

8. MTS-1804, p. 90-98 et 176-179.

9. F. Madonia, "L'esthétique de la laideur dans le Manuscrit trouvé à Saragosse", p. 185.

IO. F. Rosset, D. Triaire, De Varsovie à Saragosse, p. 113.

II. J. Potocki, Voyage dans les steppes d'Astrakhan et du Caucase, p. 154 sq. 


\section{L'image et le texte}

Dans ce passage, tiré de son Voyage dans les steppes d'Astrakhan et du Caucase (1797), Potocki se distancie des conventions du récit de voyage. Habituellement, le voyageur-narrateur fait état de sa découverte de la facticité des textes et de sa culture livresque, remises en cause par le dévoilement de l'«autre», du «réel» et du «vrai» de l'expérience immédiate et personnelle ${ }^{12}$. Le discours de Potocki n'échappe pas totalement à ces idées. La place prédominante qu'il offre aux figures de bohémiens et de nomades, dans le Manuscrit trouvé à Saragosse, semble bien traduire sa fascination pour les voyages et les déplacements ainsi que le rôle que ces derniers peuvent jouer dans la constitution d'un véritable savoir ${ }^{13}$. C'est d'ailleurs ce que Potocki semble faire dire au brigand Zoto, quand il s'adresse à Giulio Romati, personnage considéré par certains commentateurs comme une des incarnations de l'auteur dans le récit potockien ${ }^{14}$ :

Seigneur Romati, vos talents font déjà honneur à votre pays, vous lui en ferez encore plus lorsque les voyages que vous entreprenez auront étendu la sphère de vos connaissances ${ }^{15}$.

Toutefois, l'expérience du réel ne semble pas suffire à Potocki, qui n'en fait qu'une expérience au second degré. Les images des "peuples de la haute Asie» ne les font pas vivre mais revivre; elles raniment plus qu'elles convoquent la réalité, que les lectures de l'enfance et de l'âge adulte ont, bien plus et bien avant la découverte "réelle» des steppes d'Asie centrale, construite et invoquée.

Potocki, on le voit, n'est un écrivain ni néo-platonicien, ni idéaliste, et cela pour deux raisons. D'abord parce qu'il ne pense pas que l'image soit nécessairement et essentiellement trompeuse - même s'il lui arrive

I2. Sur cette question des rapports entre géographie réelle et imaginaire, voir le récent P. Bayard, Comment parler des lieux où l'on n'a pas été?.

I3. L. Fraisse, Potocki et l'imaginaire de la création, p. 91 et 267.

I4. Luc Fraisse parle, au sujet des figures de van Worden et de Romati, de «deux troncs d'où partent les récits autobiographiques des autres personnages" (Potocki et l'imaginaire de la création, p. 91). Sur cette question de l'auteur, voir F. Rosset, "Cherchez l'auteur dans le Manuscrit trouvé à Saragosse».

I5. MTS-1804, p. 243. 
souvent de l'être ${ }^{16}$. Potocki dessine d'ailleurs quand il voyage, associant souvent, dans ses textes, l'authenticité du témoignage à la sincérité du regard jeté sur lui:

Je ne promets au lecteur qu'une chose, c'est de ne pas fermer les yeux. Tout ce que j'aurai l'occasion de voir, je le raconterai ${ }^{17}$.

Ensuite et surtout parce que Potocki croit dans les puissances de vérité du langage, qu'il ne réduit pas à un art de la sophistique ${ }^{18}$. Dans cette confrontation des mots et des images, les premiers paraissent, chez Potocki, toujours sortir vainqueurs, car, plus que le pinceau, la plume permet de rendre compte de la complexité d'un réel qui n'est pas toujours réductible aux apparences d'une simple image fixe ou limitée par les conventions des arts visuels, comme le suggère Potocki, à plusieurs reprises, lors de son voyage en Asie Centrale:

Arrivé au but, je n'ai trouvé que des rives noyées et couvertes de roseaux. Et je doute que le pinceau même de Vernet pût réussir à en faire des tableaux ${ }^{19}$.

La question n'est pas seulement, ici, celle, topique, de l'indicible ou de l'ineffable, si fréquente dans les récits de voyage où il vaut toujours mieux avoir vu qu'avoir dit, quitte à être incapable de dire, et même de revenir ${ }^{20}$. Il s'agit surtout de montrer que le langage, la "langue", devrait-on dire, en se rappelant l'exemple du corps métaphorique de Pascheco, ne se construit, ne se développe qu'à mesure qu'il prend ses distances à l'égard d'une stricte et directe imitation visuelle du monde, qui n'a, en lui-même, qu'un intérêt relatif:

Mes vers sont aussi bons que des vers puissent être, et ils sont d'un usage plus général. J'ai fait de la poésie comme un instrument universel, surtout de la poésie descriptive que j’ai pour ainsi dire créée et qui me sert à décrire des choses qui d'ailleurs n'en valent guère la peine ${ }^{21}$.

I6. J. Potocki, Voyage dans les steppes d'Astrakhan et du Caucase, p. 155: «Mais aussi je rencontre de nouvelles difficultés: je vois des hommes à visages plats, qui me semblent appartenir à un même peuple; mais ces hommes parlent des langues différentes.»

I7. J. Potocki, Voyage dans les steppes d'Astrakhan et du Caucase, p. 141.

I8. Sur cette question, voir notamment B. Cassin, L'Effet sophistique.

19. J. Potocki, Voyage dans les steppes d'Astrakhan et du Caucase, p. 170.

20. G. Magherini, Le Syndrome de Stendhal.

2I. $M T S-1810$, p. 453. 
Pour Potocki, "raconter, c'est susciter une impression de déjà vu»"22; c'est même aller au-delà, en suscitant du "jamais vu», en suscitant ce qui ne peut se voir. Ce n'est qu'au prix d'un sacrifice ou d'une perte du regard - un œil crevé, chez Pascheco - que l'écrivain peut construire une langue véritablement littéraire.

\section{Le voir et le dire}

Il serait tentant, ainsi, de faire de l'art d'écrire de Jean Potocki un art exclusivement littéraire, qui aurait posé son autonomie formelle et générique en s'opposant à toutes les autres formes d'expression artistique. La situation, toutefois, est plus complexe et plus ambiguë. Le Manuscrit trouvé à Saragosse laisse en effet, je l'ai dit et j'y reviendrai dans un instant, une large place aux références à l'histoire des arts et des artistes mais aussi aux thèmes de la vision et du visible, lesquels sont étroitement liés, sous la plume de Jean Potocki, aux champs lexicaux du plaisir ou de l'attraction sexuels. Il est inutile de citer ici in extenso la rencontre et les péripéties mettant en scène Alphonse et les deux sœurs Emina et Zibeddé, lesquelles sont bien connues des lecteurs du Manuscrit et ont été largement analysées, en étant notamment mises en relation avec les théories sensualistes d'Etienne Bonnot de Condillac (1715-1780) ${ }^{23}$. La lecture de ces pages permet de montrer à quel point, pour Potocki, le regard (de celui qui voit comme de celui qui est vu) se présente toujours comme une caresse fictive. Mais, comme de bien entendu, et l'on retrouve là un thème littéralement incarné par le corps à la fois mutilé et grandiose de Pascheco, ce sentiment de plénitude et de plaisir lié à la découverte visuelle de la beauté s'accompagne presque systématiquement d'une peur ou d'une inquiétude fort ambivalente.

Ainsi, par exemple, du réveil du narrateur à Los Alcornoques, après avoir bu du vin d'Alicante et s'être aussitôt évanoui :

Non seulement je me trouvais rétabli, mais même dans un état de force et d'agitation qui avait quelque chose d'extraordinaire. La campagne me semblait émaillée des couleurs les plus vives; les objets scintillaient

22. L. Fraisse, Potocki et l'imaginaire de la création, p. 260.

23. J. Herman, "Le Traité des sensations de Potocki»; L. Fraisse, Potocki et l'imaginaire de la création, p. 221. 
à mes yeux comme les astres dans les nuits d'été, et je sentais battre mes artères surtout aux tempes et à la gorge ${ }^{24}$.

Ici, la vue du paysage suscite auprès du narrateur un véritable émerveillement, marqué par le spectacle du lustre et de l'étincelle ("émaillée", "couleurs les plus vives", "scintillaient", "astres») et par la variété des objets se présentant à sa vue. Mais cette étincelle est aussi assimilée à une flamme qui, trop forte, peut brûler et blesser, en réchauffant le sang de celui qui, comme la mouche des recueils d'emblèmes du XVI ${ }^{\mathrm{e}}$ siècle, se brûle à vouloir se rapprocher trop près de la bougie. Le plaisir de la vue est quasi irrésistible et, peut-être pour cette raison même, intensément dangereux.

D'autres passages reprennent et développent les mêmes comparaisons, comme le fameux réveil sous le gibet de Los Hermanos, dans les versions de 1804 et 1810 :

Je crus encore n'être pas bien éveillé et faire un rêve pénible. Je refermai les yeux et je cherchai dans ma mémoire où j’avais été la veille... Alors je sentis que des griffes s'enfonçaient dans mes flancs. Je vis qu'un vautour s'était perché sur moi et dévorait un des compagnons de ma couche. La douleur que me causait l'impression de ses serres acheva de me réveiller. Je vis que mes habits étaient près de moi, et je me hâtai de les mettre. [...] Comme je continuais à observer je vis près du fleuve deux voyageurs [...] Lorsque je les eus perdus de vue, je songeai à quitter mon poste ${ }^{25}$.

Enfin je me réveillai réellement; le soleil brûlait mes paupières, je les ouvris à peine, je vis le ciel, je vis que j'étais en plein air, mais le sommeil appesantissait encore mes yeux. Je ne dormais plus, mais je n'étais pas encore éveillé. Des images de supplices se succédèrent les unes aux autres, j'en fus épouvanté. Je me soulevai en sursaut ${ }^{26}$.

Dans ces deux versions, assez complexes, Potocki choisit d'entremêler plusieurs thématiques qui viennent fréquemment structurer un récit où le narrateur est présenté alternativement comme un spectateur et comme un voyeur. Le désir de voir y est associé à un danger de voir, et à une

24. MTS-1804, p. 63.

25. MTS-1804, p. 86 sq.

26. MTS-1810, p. 85. 
peur de la cécité, que l'on retrouve à plusieurs reprises exemplifiée dans le Manuscrit trouvé à Saragosse, dans la description, déjà évoquée, de Pascheco, mais aussi dans le récit que la créature livre des origines de sa mutilation:

Alors je sentis qu'un des pendus me saisissait par la cheville du pied gauche. Je voulus m'en débarrasser mais l'autre pendu me coupa le chemin. Il se présenta devant moi, faisant des yeux épouvantables et tirant une langue rouge comme du fer que l'on sortirait du feu. Je demandai grâce, ce fut en vain. D'une main, il me saisit la gorge et de l'autre il m'arracha l'œil qui me manque. A la place de mon œil, il entra sa langue brûlante. Il m'en lécha le cerveau et me fit rugir de douleur ${ }^{27}$.

Le cortège de métaphores déployé par Potocki semble s'inscrire dans la droite ligne des observations que nous avons pu faire autour du corps de Pascheco, tout en demeurant dans une ambivalence comparable. Car s'il est vrai que la "langue" d'un des pendus - pour ne pas dire, une nouvelle fois, sa «langue bien pendue»-se présente comme une arme et un danger, "rouge comme du fer que l'on sortirait du feu " dès lors qu'elle se trouve au contact de l'œil, le récit de Potocki est aussi celui d'une véritable (mise en) relation entre l'œil de Pascheco et la langue du pendu, dont la description tient à la fois, dans un esprit qui n'est pas éloigné de certains récits walpoliens ou d'épisodes sadiens, d'une infernale scène de torture et d'une relation sexuelle inavouée ${ }^{28}$. Une scène qui peut tout aussi bien être interprétée comme l'allégorie à peine voilée de la puissance dévastatrice d'une Langue à laquelle rien ne résiste que comme la métaphore des relations ambiguës et étroites que le voir et le dire entretiennent, faits de baisers et de mutilations réciproques.

\section{Fragments et textures}

Ce que le corps de Pascheco semble nous montrer et nous dire, ce n'est donc pas tant la stricte victoire de la langue, du texte et du dire sur l'œil,

27. MTS-1810, p. 96 sq.

28. Sur ces sources, voir J. Finné, «Jean Potocki et le Gothic Novel»; J. Fabre, Idées sur le roman, de Madame de Lafayette au marquis de Sade; H. van Gorp, "Le Manuscrit trouvé à Saragosse et le roman gothique». 
l'image et le voir, que l'état permanent de rivalité dans lequel ces deux formes d'expression sont constamment placées l'une par rapport à l'autre dans l'esprit de Jean Potocki. Si ce dernier croit fondamentalement aux puissances heuristiques du langage littéraire, il sait aussi sa langue hantée par les images, qu'elles soient issues du monde réel, tel qu'il a pu le rencontrer au cours de ses nombreux voyages, ou de l'histoire de l'art, auquel le Manuscrit trouvé à Saragosse ne cesse de faire référence.

J'en veux pour preuve deux récits, où la présence et la mention des œuvres d'art occupent une place centrale, et qui se trouvent enchâssés dans le récit, rapporté par Giulio Romati, de la découverte, un soir d'orage, du château en ruines de la mystérieuse princesse de Monte Salerno ${ }^{29}$ :

L'intérieur de la maison n'était pas d'une chaumière. On y voyait belles tentures de Flandres à personnages si bien ouvrés et portrait qu'ils semblaient vivants, des lustres à bras en argent fin et massif, de riches cabinets en ivoire et ébène, des fauteuils en velours de Gênes, garnis de franges d'or et un lit en moire de Venise ${ }^{30}$.

Par exemple, dit la dame, je vous permets d'admirer la pièce. Je l'admirai en effet. Le parquet était en effet en lapis, incrusté de pierres dures en mosaïque de Florence, dont une table coûte plusieurs années de travail. Le dessin avait une intention générale et présentait l'ensemble le plus régulier. Mais lorsqu'on en examinait les divers compartiments, on voyait que la plus grande variété dans les détails n'ôtait rien de l'effet que produit la symétrie. En effet, quoique ce fût toujours le même dessin, ici il offrait l'assemblage des fleurs les plus nuancées, là c'étaient les coquillages les mieux émaillés, plus loin des papillons, ailleurs des colibris. Enfin les plus belles pierres du monde étaient employées à l'imitation de ce que la nature a de plus beau. Au centre de ce magnifique parquet, était représenté un écrin composé de toutes les pierres de couleur, entouré d'un fil de grosses perles. Le tout paraissait en relief et réel comme dans les tables de Florence ${ }^{31}$.

Ces deux exemples, que je me permets ici de citer entièrement afin de restituer l'énumération des différents objets aperçus par le narrateur,

29. MTS-1804, p. 246-262.

30. MTS-1810, p. 203.

31. MTS-1810, p. 241. 
rendent bien compte de la volupté dans laquelle le narrateur et l'écrivain se complaisent volontiers en dressant l'inventaire long et détaillé des éléments constitutifs du spectacle auquel ils assistent. En offrant au narrateur la compagnie de la dame d'honneur de la propriétaire des lieux, Potocki semble d'ailleurs signifier, par associations d'idées, le plaisir pris dans l'acte de voir et de toucher du regard, mais aussi, plus vraisemblablement encore, vouloir rendre compte des dangers potentiels de ce regard. Mais ces deux récits, où la langue de l'écrivain le dispute au métier de l'artisan, renvoient à un autre aspect décisif de la comparaison du "voir » et du "dire " chez Potocki: la dimension fragmentaire et éclatée de la représentation. Luc Fraisse a eu raison de souligner que le "parquet en lapis", mentionné dans le premier extrait cité, pouvait être considéré comme la métaphore vivante de la trame d'un texte dont la logique ne se dévoile que progressivement et partiellement au lecteur, qui découvre "peu à peu que le récit complexe à travers lequel il progresse répond à une intention générale " 32 . Il semble même possible de généraliser cette interprétation à une grande partie des œuvres d'art visuelles convoquées par Potocki, qui, dans un écrit politique daté de 1790, compare l'art des administrateurs à celui qui consiste à fabriquer une image cohérente en juxtaposant différentes tessères: "lorsqu'il veut raccommoder son tableau, il [le peintre en mosaïque] retire habilement quelques-uns des mille cubes vitrifiés qui le composent, et en met d'autres à leur place» ${ }^{33}$. La métaphore employée ici par Potocki, laquelle renvoie au métier du mosaïste, du peintre et du tisserand, mais peut tout aussi bien évoquer sa propre conception de l'écriture, montre que, pour l'auteur du Manuscrit trouvé à Saragosse, la logique d'un récit est moins du ressort de la "construction" intellectuelle, effectuée a priori, que d'une sorte de "bricolage», au sens que Claude Lévi-Strauss a donné à ce mot dans la Pensée sauvage (1962), où "la composition de l'ensemble n'est pas en rapport avec le projet du moment, ni d'ailleurs avec aucun projet particulier, mais est le résultat contingent de toutes les occasions qui se sont présentées de renouveler ou d'enrichir le stock [des matériaux utilisés], ou de l'entretenir avec les résidus de constructions et de destructions antérieures" ${ }^{34}$.

32. L. Fraisse, Potocki et l'imaginaire de la création, p. 92.

33. J. Potocki, Essay d'aphorismes sur la liberté, p. 310.

34. C. Lévi-Strauss, La Pensée sauvage, p. 27. 


\section{Imitations et dépassements}

Pour Potocki, l'art de l'écrivain se situe donc toujours en rapport avec celui de l'artiste, au sens où il doit, tout à la fois, s'en inspirer et le dépasser. S'en inspirer, tout d'abord, puisque l'œuvre d'art - un tableau, un tapis, une mosaïque, un tissu - fournit à l'écrivain le modèle d'une production artistique fondée sur une juxtaposition de couches successives, sur une accumulation de petits éléments disparates qui créent, par superpositions et ajouts successifs, une texture qui constitue la trame même de l'écriture. Chez Potocki, ce modèle d'un récit littéraire, qui ne cache pas être fait "de bric et de broc» et valorise même cette conception atomisée et feuilletée de la construction narrative, n'est d'ailleurs pas seulement théorique mais pratique. L'auteur du Manuscrit trouvé à Saragosse ne dissimule pas en effet que le récit qu'il rapporte de Giulio Romati est directement inspiré d'un "gros volume, écrit en caractères gothiques, dont le titre était Relations curieuses de Happelius", un ouvrage qui a réellement existé, écrit par le savant Eberhard Werner Happel (1647-1690), et dont la première et la seule édition connue a été publiée à Hambourg entre 1683 et $1685^{35}$. Potocki le fait préciser dans la version de 1810 :

Ici, j'interrompis le chef [Giulio Romati] pour lui dire que j'avais feuilleté chez le cabaliste [don Pedre de Uzeda] les relations variées de Happelius, et que j'y avais trouvé une histoire à peu près semblable. Cela peut-être, reprit le chef, peut-être Romati a-t-il pris son histoire dans ce livre. Peut-être l'a-t-il inventée. Toujours est-il que son récit contribua beaucoup à me donner le goût des voyages ${ }^{36}$.

Cet effet de télescopage et de papillotage des sources, réelles et/ou imaginaires, qui s'emboitent les unes dans les autres, sera d'ailleurs curieusement renforcé, après la rédaction des deux versions du Manuscrit, par Pierre-Marie-Jean Cousin de Courchamps (1783-1849) qui, dans ses apocryphes Souvenirs de la marquise de Créquy (1834), plagiera lui-même

35. Son titre complet est E. G. Happelii grösste Denckwürdigkeiten der Welt, oder Sogenannte relationes curiosae, worinnen dargestellet und nach dem Probier-Stein der Vernunft examiniret werden, die vornehmsten physicalische, mathematische, historische und andere merckwürdige Seltzahmkeiten welche an unserm sichtbahren Himmel, in und unter der Erden und im Meer jemahlen zu finden oder zu sehen gewesen und sich begeben haben, der 1. [-anderer] Theil, Hambourg, T. von Wiering, 1683-1685.

36. MTS-1810, p. 251 sq. 
le récit de Romati en l'augmentant d'une série de nouvelles descriptions et en prêtant aux lieux décrits une existence réelle... ${ }^{37}$

Pour Potocki, le «dire» est donc toujours une autre façon de "voir", dans la mesure même où le modèle littéraire auquel il s'astreint est dominé par le refus de la linéarité et l'affirmation de la polyphonie des modèles et des genres. Dans ce cadre, effectivement, "l'effet produit par un récit a plus d'importance que son origine (et en ce sens que son originalité) " ${ }^{38}$. De fait, dans le Manuscrit trouvé à Saragosse, la langue a toujours, si j'ose dire, le dernier mot, manipulant les références visuelles exactement de la même manière que les sources textuelles. C'est en cela, sans doute, que, pour Potocki, la langue demeure systématiquement première, dépassant le modèle rival de l'image par son inclusion au sein même de la texture et des transformations propres à son texte. Cette force spécifiquement heuristique de la langue, qui la rend supérieure à toute forme d'expression poétique, y compris visuelle, explique l'attachement de Jean Potocki, quand il cite des artistes et des ouvres d'art, à brouiller les cartes et les pistes. En guise d'exemple, je citerai ici un passage dans sa totalité, dans la mesure où les différentes références me semblent liées les unes aux autres dans la structure du discours:

Je levais donc les yeux et ils tombèrent d'abord sur un tableau de Raphaël qui paraissait être la première idée de son Ecole d'Athènes et qui était plus beau par le coloris, d'autant qu'il était peint à l'huile. Ensuite je remarquai un Hercule aux pieds d'Omphale; la figure de l'Hercule était de Michel-Ange, et l'on reconnaissait le pinceau du Guide dans la figure de la femme. En un mot, chacun des tableaux de ce salon était plus parfait que tout ce que j'avais vu jusqu'alors. La tapisserie était un velours vert tout uni dont la couleur faisait ressortir les peintures. Aux deux côtés de chaque porte étaient des statues un peu plus petites que nature. Il y en avait quatre. L'une était le célèbre Amour de Phydias [sic], dont Pythagore exigea le sacrifice; la seconde, le Faune du même artiste; la troisième, la véritable Vénus de Praxitèle, dont celle de Médicis n'est qu'une copie; la quatrième, un Antinoüs de la plus grande beauté ${ }^{39}$.

37. P.-M.-J. Cousin de Courchamps, Souvenirs de la marquise de Créquy, t. III, p. 335. Sur ce point, voir L. Fraisse, Potocki et l'imaginaire de la création, p. 93.

38. L. Fraisse, Potocki et l'imaginaire de la création, p. 147.

39. MTS-1810, p. 242 sq. 
Cet inventaire, remarquablement complet, est aussi étrangement, et presque systématiquement biaisé par rapport à la réalité historique. On y trouve deux mythiques sculpteurs grecs, Phidias (vers 490-430 av. J.-C.) et Praxitèle (vers 400-326 av. J.-C.), deux artistes italiens de la Renaissance, Raphaël (1483-1520) et Michel-Ange (1475-1564), et un autre du début du XVIIe siècle, Guido Reni, dit le "Guide» (15751642). Certaines des œuvres évoquées existent bel et bien comme la Vénus Médicis et l'Antinoüs du Belvédère; d'autres s'inspirent, plus ou moins librement, d'ouvrages réels, comme l'Amour et le Faune de Phidias (Potocki fond et confond ici, peut-être volontairement, plusieurs antiques différentes), le modello peint à l'huile de l'Ecole d'Athènes (qui n'existe pas, mais évoque peut-être le grand dessin préparatoire conservé à la Biblioteca Ambrosiana, à Milan), ainsi que l'Hercule et Omphale peint conjointement par Michel-Ange et Reni, ce qui est impossible puisque le second est né après la mort du premier, et qui renvoie probablement au plus célèbre des tableaux illustrant ce thème, celui de Peter Paul Rubens (1577-1640), conservé au Louvre.

L'ensemble de ces décalages et écarts par rapport à la vérité des dates et des faits est bien sûr entièrement voulu, dans la mesure où ces références sont davantage poétiques qu'historiques. La fiction y joue un rôle essentiel, orchestrée par un auteur qui connaît trop bien ses classiques - qu'il cite à foison, ne serait-ce que pour entretenir avec son lecteur un rapport mêlé de complicité et d'érudition - pour savoir quand il ne fallait point commettre d'erreurs (la Vénus Médicis), et quand, au contraire, il s'agissait d'échafauder des chimères afin d'exciter l'imagination du spectateur (l'impossible et introuvable Hercule et Omphale). Par les ressources littéraires qu'il déploie, Potocki ne se contente pas de marquer la force et la puissance du mot, capable de recréer ce que l'histoire n'a pas pu ou voulu fabriquer, renouant ainsi avec les anciens débats sur les mérites et les pouvoirs respectifs des différents arts ${ }^{40}$. Il refabrique ces œuvres, en les déplaçant d'un médium à un autre (de la fresque à l'huile, chez Raphaël), d'un artiste à l'autre (l'Amour et le Faune, de Praxitèle à Phidias), voire d'une œuvre à une autre (la Vénus de Praxitèle et sa copie, la Vénus Médicis). Pour cela, il lui faut jouer autour de références comprises et partagées par ses lecteurs les plus cultivés, et, jouant de la

40. Voir à ce sujet J. H. Hagstrum, The Sister Arts; L. Fallay d'Este (éd.), Le Paragone; R. Preimesberger, Paragons and Paragone. 
surprise ou de la confusion, dérouter et mettre en défaut des horizons d'attente trop fermement fixés autour d'une culture canonique héritée de la lecture des grands recueils de l'histoire de l'art.

$\mathrm{Au}$ terme de ce bref parcours dans quelques-unes des arcanes du Manuscrit trouvé à Saragosse, où les noms de Velázquez et de Pacheco côtoient ceux de Raphaël ou de Michel-Ange, où peintures et ruines, mosaïques et tapisseries semblent reconstituer l'univers visuel et onirique de Jean Potocki, il me semble désormais clair que, pour l'auteur polonais, les œuvres d'art valent moins par elles-mêmes que pour leur force poétique et mythique, au sein d'un univers littéraire où elles sont convoquées et soumises aux désirs d'une langue qui les refabrique. Potocki, en cela, ne fait pas seulement œuvre de traducteur, au sens large du terme, concevant son texte comme un immense champ d'expériences et d'expérimentations sur la langue elle-même, ses sources, ses genres et ses styles; il s'institue aussi comme un créateur, au sens démiurgique du terme, qui revisite l'histoire de l'art en fonction des situations qu'il a lui-même imaginées mais aussi au gré des caprices et des fantasmes des personnages hantant et visitant son récit à tiroirs. Une fête pour l'œil, donc, mais aussi une fête pour la langue... 


\section{BIBLIOGRAPHIE}

\section{Textes}

Cousin de Courchamps, Pierre-Marie-Jean, Souvenirs de la marquise de Créquy, 7 vols, Paris, Fournier Jeune, 1834-1835.

Delacroix, Eugène, Journal, 1822-1863, éd. par André Joubin, Paris, Plon, 1987.

MTS-1804 et MTS-1810 = Pотоскі, Jean, Manuscrit trouvé à Saragosse (version de 1804 et version de 1810), éd. par François Rosset, Dominique Triaire, Paris, Flammarion, 2008 (GF 1342-1343).

Pотоскі, Jean, Voyage dans les steppes d'Astrakhan et du Caucase, in Euvres, t. 2, éd. par François Rosset, Dominique Triaire, Louvain, Peeters, 2004.

_, Essay d'aphorismes sur la liberté, in Euvres, t. 3, éd. par François Rosset, Dominique Triaire, Louvain, Peeters, 2004.

\section{Travaux}

Bayard, Pierre, Comment parler des lieux où l'on n'a pas été?, Paris, Editions de Minuit, 2012.

CAssin, Barbara, L'Effet sophistique, Paris, Gallimard, 2001.

FABre, Jean, Idées sur le roman, de Madame de Lafayette au marquis de Sade, Paris, Klincksieck, 1979.

Fallay D’Este, Lauriane (éd.), Le Paragone: le parallèle des arts, Paris, Klincksieck, 1992.

Finné, Jacques, "Jean Potocki et le Gothic Novel», Revue des langues vivantes, XXXVI (1970-1972), p. 141-165.

Fraisse, Luc, Potocki et l'imaginaire de la création, Paris, Presses de l'Université Paris-Sorbonne, 2006. 
Hagstrum, Jean H., The Sister Arts: The Tradition of Literary Pictorialism and English Poetry from Dryden to Gray, Chicago, University of Chicago Press, 1987.

Herman, Jan, "Le Traité des sensations de Potocki", in Le Manuscrit trouvé à Saragosse et ses intertextes. Actes du colloque international, Leuven-Anvers, 30 mars - $1^{e r}$ avril 2000, éd. par Jan Herman, Paul Pelckmans, François Rosset, Louvain, Peeters, 2001, p. 219-229.

Herman, Jan, Pelckmans, Paul, Rosset, François (éds), Le Manuscrit trouvé à Saragosse et ses intertextes. Actes du colloque international, Leuven-Anvers, 30 mars - $1^{\text {er }}$ avril 2000, Louvain, Peeters, 2001.

Lévi-Strauss, Claude, La Pensée sauvage, Paris, Plon, 1962.

Madonia, Francesco, "L'esthétique de la laideur dans le Manuscrit trouvé à Saragosse", in Le Manuscrit trouvé à Saragosse et ses intertextes. Actes du colloque international, Leuven-Anvers, 30 mars $1^{\text {er }}$ avril 2000, éd. par Jan Herman, Paul Pelckmans, François Rosset, Louvain, Peeters, 2001, p. 179-188.

Magherini, Graziella, Le Syndrome de Stendhal: du voyage dans les villes d'art, Paris, Usher, 1990.

Moussa, Sarga, "Le nomadisme chez Potocki: des récits de voyages au Manuscrit trouvé à Saragosse", Revue de littérature comparée, CCLXXXVII/3 (1998), p. 231-353.

Preimesberger, Rudolf, Paragons and Paragone: Van Eyck, Raphael, Michelangelo, Caravaggio, Bernini, Los Angeles, Getty Research Institute, 2011.

Rosset, François, "Cherchez l'auteur dans le Manuscrit trouvé à Saragosse", Vives lettres, V (1998), p. 39-60.

Rosset, François, Triaire, Dominique, De Varsovie à Saragosse. Jean Potocki et son cuvre, Louvain, Peeters, 2000.

van Gorp, Hendrik, "Le Manuscrit trouvé à Saragosse et le roman gothique", in Le Manuscrit trouvé à Saragosse et ses intertextes. Actes du colloque international, Leuven-Anvers, 30 mars $-1^{\text {er }}$ avril 2000, éd. par Jan Herman, Paul Pelckmans, François Rosset, Louvain, Peeters, 2001, p. 237-245. 
